\title{
Comparison of emergency chest ultrasound with radiography in the diagnosis of community-acquired pneumonia in Emergency Department
}

\author{
Nikoletta Daoulari* \\ Emergency Department of Brugmann Hospital, Brussels Belgium
}

*Correspondence to: Nikoletta Daoulari, Emergency Department of Brugmann Hospital, Brussels Belgium, E-mail: nikolettadaoulari@gmail.com Received: October 24, 2019; Accepted: October 31, 2019; Published: November 03, 2019

\section{Introduction}

Community-acquired pneumonia, with clinical signs fever, dyspnea and cough, productive or not, is a common problem and one of the most common causes for admission in Emergency department, mainly for elderly people with multiple comorbidities. It is a common and serious infectious disease associated with high morbidity and mortality. Pneumonia is the sixth leading cause of death and the most common infectious disease in the world. Early and effective antibiotic treatment is important. Therefore, a correct and quick diagnosis is required. The diagnosis is mainly based on history and physical examination, but for some patients the diagnosis is difficult especially in demented or disabled patients or in serious situations requiring urgent intervention. However, the diagnosis is often wrong even nowadays. Currently, the chest X-ray (CXR) is recommended for routine evaluation of a patient suspected with pneumonia because the history and physical examination alone cannot provide certainty in this diagnosis. Some studies have shown that diagnostic accuracy of chest X-ray is suboptimal. The sensitivity of this method, according to the studies considered, varies from 73 to $88 \%$. In the emergency, due to the workload taking chest X-ray may be delayed or of poor quality, either because it is in lying position or because the patient is not very cooperative. Thoracic CT (CT) is considered the "gold-standard" but is not often feasible in an emergency. As the chest CT is more irradiating, its use in the diagnosis of pneumonia is contraindicated in infants and pregnant women [1-6].

To counter this paradox, which wants the most critical patient to have the least access to modern techniques, the clinician would like to have a means to solve the main problem of emergency: combine speed and reliability. Such a method does it exist for the diagnosis of pulmonary infection? Ultrasound is a fast, non-invasive method of investigation frequently used in Emergency Department. Several studies have shown that ultrasound at the patient's bedside could assist in the diagnosis of cardiogenic pulmonary edema, pneumonia, pneumothorax or pulmonary embolism. It is a method both old and recent, since its first medical applications date back to 1946, while paradoxically, it is only recently that its potentialities in the emergency department have been recognized (Annex 1) [7-10].

The purpose of our study is twofold

1. Estimate the performance of ultrasound as an aid to the diagnosis of pneumonia and to compare it with the performance of chest X-ray in the Emergency department

2. Estimate if lung ultrasound can become the tool of choice for the diagnosis of pneumonia and replace the chest X-ray in the future

\section{Material and method}

This is a prospective study, carried out by a single operator for a period of 3 months, between January 01, 2019 and March 30, 2019. Patients presenting to the Emergency Department of the Brugmann Hospital, Brussels, Belgium, with a suspicion of infectious pneumonia were included. The study protocol was approved by the Ethics Commission of Brugmann Hospital and a written informed consent was obtained from all included patients. Informed consent was obtained from the patient or a person close to the patient if the patient was not able to give it.

\section{Inclusion criteria}

Patients over the age of 18 with compatible signs and symptoms of a pulmonary infection were studied. According to international guidelines, suggestive clinical elements were cough, fever, dyspnea, sputum and pleural chest pain. In the elderly ( $>75$ years), an altered mental state and general state degradation were also taken into account [11-14].

\section{Exclusion criteria}

Patients under 18 and pregnant women were excluded.

\section{Recruitment of data}

Eligible patients first received pulmonary ultrasound at their admission at ER.Ultrasound was performed by the same emergency physician trained in ultrasound emergency, having performed at least 25 prior pulmonary ultrasounds. The device used, Exagyne ECM, was equipped with a $3.5-5 \mathrm{MHz}$ convex network probe. The results were consistent with the international literature as described the normal and pathological lung. In the normal lungs, the axillary lines separate the anterior, lateral and posterior walls, each of these regions can be divided into quadrants. At the bedside of the patient, the probe was placed perpendicularly, obliquely and parallel to the ribs of the anterior, lateral and posterior thorax. In agreement with the literature, each hemithorax was divided into 5 zones: 2 anterior, 2 lateral, 1 posterior [15-17] (Figure 1). 


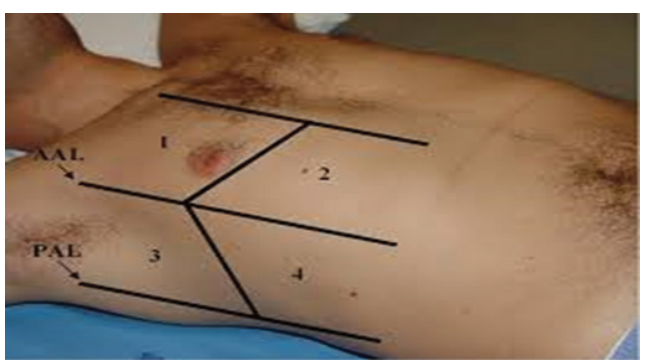

Figure 1: The areas of thoracic ultrasound considered in the study. Zones 1 and 2: anterior superior and anterior; zones 3 and 4: lateral superior and lateral basal. Each zone was the same on the right and on the left. All rights Volpicelli and all [17].

The first step, to avoid many confusions, is the separation of the thorax from the abdomen: the diaphragm should be spotted first. The superficial layers of the thorax consist of subcutaneous tissue and muscle. The ribs, in longitudinal scan, appear at the form of curvilinear structures associated with a posterior acoustic shadow. The pleura looks like an echogenic line showing continuous intrinsic motion during the breathing called "Lung sliding sign". The parenchyma, filled with air, prevents any ultrasound visualization under the line of the pleura. However, the wide difference in acoustic impedance between the pleura and the parenchyma creates typical horizontal artifacts. These are a series of echoic parallel lines at a distance equal to another line originating from the pleural line These artifacts have been defined "A lines "by Lichtenstein [18]. Other vertically oriented artifacts, "comet tails" (B lines) (according to Lichtenstein [7] and Volpicelli [17]) may be present (Figure 2). The "B lines" which come from the pleural lung interface and reach the edge of the screen, clear the "A lines' and moves with 'lung sliding' and is absent in the normal lung [7]. This artefact is the result of close contact between air (lung) and water, two elements having a very distant acoustic impedance, the water transmitting sounds well, while the air stops them.
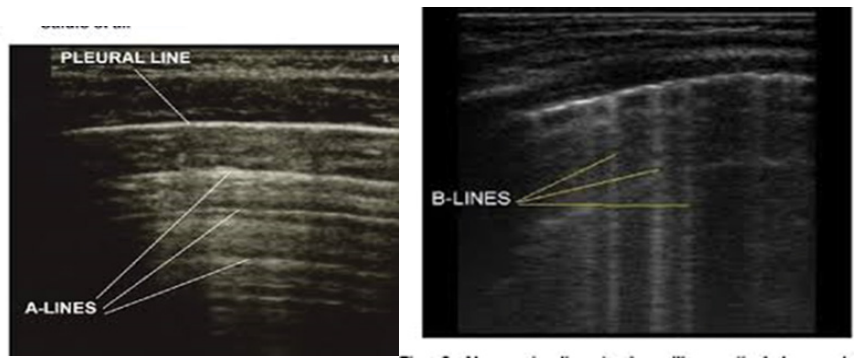

Figure 2: (left) normal lung. Note the echogenic line representing the pleura line and the horizontals artifacts, called lines A. (right) Abnormal lung. Discrete laser line vertical hyperechoic reverberation artifacts which come from the pleural line, B lines.
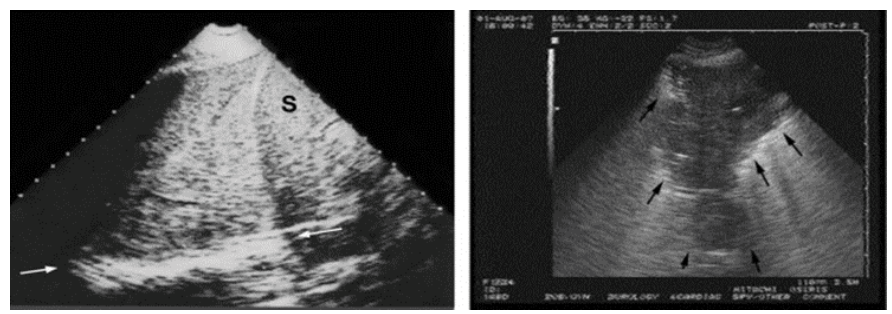

Figure 3: Two signs of lung consolidation. Left: Massive consolidation (PLAPS probe) invades the entire lower left lobe. No aerated lung tissue is present and no fractal signs can be generated. The deep border is at the mediastinal line (arrows). The pattern looks like a tissue, similar to the spleen (S). The thickness of this image is about $10 \mathrm{~cm}$, value incompatible with a pleural effusion. Right: consolidation of the middle lobe, which does not invade the entire lobe. This generates a jagged fractal boundary between the consolidation and the underlying aerated lung (arrows): the very specific chipping (or fractal) sign. Such anterior consolidation generates profile C in the BLUE protocol [18].
Other ultrasound findings related to pneumonia include abnormalities in pleural line of coarse appearance, obviously associated with the abolition of 'lung sliding', explained by inflammatory adhesions due to exudates. In addition, the detection of alveolar disorders (regardless of their number and size) on the anterior wall defines the consolidation, and indicates as a priority the diagnosis of pulmonary infection at the origin of the acute respiratory failure.

In addition to bedside ultrasound, all subjects underwent X-rays thoracic in bed. Inflammatory syndrome, leukocytosis and CRP, were sought in all the patients. Blood cultures, sputum culture and serology were also requested. X-rays were read by a resident radiologist of the department, ignoring the results of the ultrasound. The protocol, mentioning the presence or absence of a consolidation or the presence of pleural effusion or vascular overload causing an alternative diagnosis, such as acute pulmonary edema explaining the symptomatology of patients, was compared to the ultrasound protocol that had been established without knowledge of the radiological protocol. As community-acquired pneumonia was defined each patient with characteristics clinical manifestations of pulmonary infection, including cough, pleural pain, fever, sputum production and dyspnea with physical examination suggesting pneumonia (crackles, rattles). Hospitalized patients were followed to collect the results of blood cultures and sputum as well as to be aware of their clinical course. The final diagnosis was also collected and compared with the diagnosis in the emergency department.

The questions posed by this study were

- $\quad$ The sensitivity and specificity of pulmonary ultrasound are comparable with those of chest X-ray for the diagnosis of communityacquired pneumonia in Emergency department?

- Would it be possible to use pulmonary ultrasound as an alternative examination in patients presenting for suspicion of pulmonary infection?

- Could this test be used only in pregnant women and in children to avoid exposure to harmful radiation?

\section{Results}

We studied 45 patients who were diagnosed with pneumonia in the emergency room based on history and clinical examination .26 (57.5\%) women and 19 (42.5\%) men of middle age of 64.2 years old. All patients benefited a chest X-ray and ultrasound pulmonary. All patients were hospitalized. No patient was transferred to intensive care unit. Follow-up has always been associated with diagnosis, showing an improved clinical condition (no fever, cough or dyspnea) and a decrease in inflammatory laboratory indices such as white globules and CRP. During hospitalization, pneumonia was confirmed in 37 patients (82\%). Among the other patients (17.77\%), 5 influenza were diagnosed, as well as one tuberculosis and 2 cardiac decompensations.

Of the 37 patients with a final diagnosis of pneumonia 27 (73\%) had a positive chest X-ray and 10 (27\%) had an X-ray that wasn't show a pulmonary infection. In addition, 5 (62.5\%) patients diagnosed with another affection $t$ had a normal X-ray and 3 (37.5\%) an X-ray with a suspicious consolidation or pleural effusion. On the other hand, of the 37 patients with confirmed pneumonia, the ultrasound was positive at 35 (94\%) (With an image of alveolar disorders (10) or with unilateral B lines (19) or both (6). Of 37 patients with pneumonia, just 2 (5\%) had a normal ultrasound.

From the 8 patients with another diagnosis, 5 (62\%) had a normal 


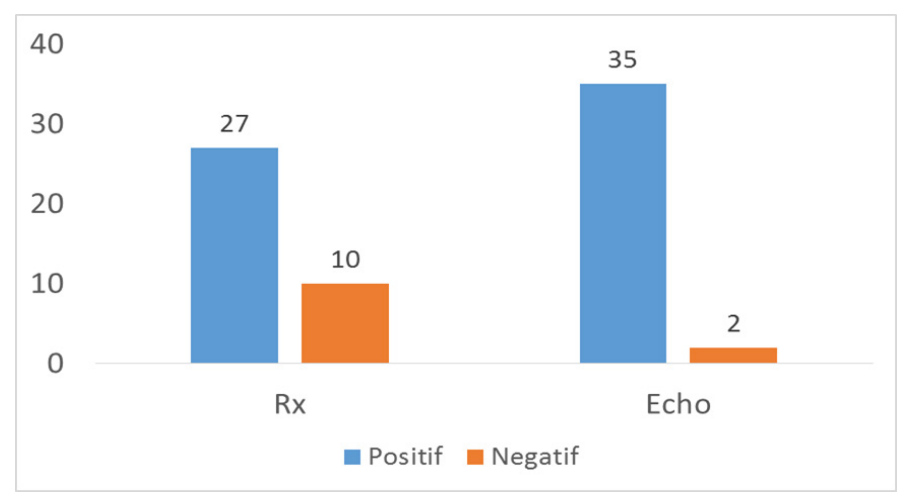

Chart 1: Shows the results of radiography and lung ultrasound in patients with confirmed pneumonia at their exit of hospital.

ultrasound and 3 (37.5\%) an ultrasound suspect of pulmonary infection with two of them having the presence of lines B but bilateral, sign of cardiac decompensation. In all 8 patients, the results of the ultrasound and chest X-ray were perfectly concordant (Tables $\mathbf{1}$ ).

\begin{tabular}{|c|c|c|}
\hline & Rx abnormal & Rx normal \\
\hline Echo abnormal & 3 & 0 \\
\hline Echo normal & 0 & 5 \\
\hline
\end{tabular}

Table 1: Results of radiography and ultrasound in patients

Not suffering from pneumonia

Of the 37 patients diagnosed with pneumonia, 67.6\% (25) of patients had signs related to pneumonia on ultrasound, but also at chest X-ray. 27\% (10) had signs on the ultrasound but not on the radio. $5.4 \%$ (2) of the patients had results in X-ray but not on ultrasound. We did not find anyone with pneumonia with a normal chest X-ray and ultrasound.

This study shows that the sensitivity of ultrasound in the diagnosis of pneumonia is $94.6 \%$ with a positive predictive value at $92.09 \%$ compared to the sensitivity of the chest X-ray that was at $73 \%$ and its positive predictive value at $89.9 \%$. There was no difference for the specificity of the two methods which was at $62.5 \%$ for both to exclude community-acquired pneumonia but the negative predictive value was higher for ultrasound (71.48\%) than for chest X-ray (33.39\%) leaving $28.52 \%$ and $66.61 \%$ chance respectively that it is false negative.

The McNemar test determined that there was a statistically significant difference between the two methods (echo and radio) ( $\mathrm{p}=$ 0.043 ) but the number of cases with positive ultrasound and negative chest X-ray is significantly greater than the number of patients with negative ultrasound and positive radiography.

The results are given in Table 2.

\begin{tabular}{|c|c|c|}
\hline & RX positive & Rx négative \\
\hline Echo positive & 25 & 10 \\
\hline Echo négative & 2 & 0 \\
\hline
\end{tabular}

Table 2:

\section{Discussion}

Pneumonia is a common and serious infectious disease and is often misdiagnosed, even nowadays. It is responsible for significant morbidity and mortality in the whole world. Early and effective antibiotic treatment is important. Currently, the lung radiography is the imaging modality most often used to diagnose communityacquired pneumonia, pleural effusion or cardiac decompensation to patients admitted to the emergency department with symptoms of respiratory failure. The findings such as consolidation, peri-bronchial nodules (bronchopneumonia), opacity or random nodules are compatible with pneumonia. Although radiography can be used at the bedside, it only provides limited information especially if the patient is not collaborated or in a lying position which ruins the quality and the interpretation of radiography. The scanner is considered the gold standard for the diagnosis of pneumonia but it exposes the patient to significant radiation. Moreover, it cannot be realized at patient's bedside and its cost is very high. It has been known since long time that ionizing radiation increases the risk of cancer. Children are more often victims of cancer than adults because they havecells that divide faster than adults and have a longer life expectancy [19-21]. In addition, exposure of pregnant women to $\mathrm{X}$-rays is avoided as much as possible. The use of bedside ultrasound for pulmonary examination is accepted by a growing number of doctors and some examination protocols have been developed [22-25]. Ultrasound is gaining more and more ground for diagnosis in the emergency department. We use it for different diagnoses (FAST echo for trauma, echo cardiac for the image of 4 cavities and search for pericardial effusion). It is also used for the examination of the aorta when suspected rupture of aortic aneurysm. Ultrasound is also widely used for the placement of central catheters and is used as an aid when performing locoregional anesthesia. Several studies have shown that bedside ultrasound is useful for diagnosis of cardiogenic pulmonary edema $[26,17,27,28]$ and that ultrasound is more specific than the radio to diagnose a pneumothorax $[8,29,30]$.

Zhang $\mathrm{M}$ report that the average time required to detect pneumothorax by ultrasound was much more short than that of radio [28]. Ultrasound in the patient's bed is also a non-invasive test for the diagnosis of pulmonary embolism [29-30]. Cortellaro reports that the sensitivity of standard radiography to diagnose pneumonia was at $69 \%$, while that of bed ultrasound was well above 96\% [9]. Parlamento reports that the standard radiography can diagnose $75 \%$ of pneumonia cases, while ultrasound can be diagnosed in $96 \%$ of cases. Lichtenstein found that thedynamic air bronchogram can distinguish pulmonary consolidation from atelectasis [31-33].

It is possible that a chest CT scan or an ultrasound scan shows a pulmonary consolidation even when the chest X-ray shows no obvious abnormality (Appendix 2). This could be attributed to the superposition of images on an X-ray film, in which the heart, mediastinum or diaphragm may partially obscure the lung lesions. In comparison, the ultrasound can be used to make the image of each intercostal space, providing more information than radio and contributing to the diagnosis of radio-negative patients with pneumonia. Most researchers use the detection of pulmonary consolidation for diagnose pneumonia on the patient's bedside ultrasound $[9,33,25]$. In a recent multicenter research, Reising indicates that ultrasound was sensitive in $93.4 \%$ of cases and specific in $97.7 \%$ for the diagnosis of pneumonia and $86.7 \%$ of patients with pneumonia had air bronchogram [33]. Consolidation is an common finding in ultrasound, but not always present. 'Blue protocol' means that the $\mathrm{B}$ lines are correlated with a interstitial and focal edema (pneumonia, pulmonary contusion) or diffuse (acute respiratory distress syndrome, cardiogenic pulmonary edema). This is a interstitial syndrome close to the $\mathrm{B}$ profile, with the difference that lung sliding is here abolished. This profile is called B 'profile. The priority diagnosis is pneumonia. In this study, the focal interstitial pattern and consolidation accounted for $64 \%$ and $43 \%$ sensitivity for the diagnosis of pneumonia. Xiao-Lei shows that the focal interstitial model may be a sign of pneumonia but not specific. Subpleural lesions may also 
occur in cases of pneumonia. In our study, pulmonary ultrasound can diagnose $94.5 \%$ of pneumonia in patients admitted in the emergency department on suspicion of pneumonia while the radiography will detect that $73 \%$ of cases. With ultrasound only the $5.4 \%$ of diagnosis of pneumonia was lost, whereas with X-ray $27 \%$ of pneumonias were not detected. At the end, the ultrasound and X-ray detected the same number of patients without pneumonia $(62.5 \%)$ despite being admitted on suspicion of pneumonia $[33,34]$.

\section{Limitations}

Our study also presented limitations. Ultrasound may lack consolidations which do not reach the pleura. The presence of ultrasound anomalies not revealed by radio has not been confirmed by a gold standard such as the chest scanner, which cannot systematically be uses for obvious ethical reasons. Another limitation of our study is that only one operator, trained in ultrasound application emergency departments with at least 25 pulmonary ultrasounds, performed all ultrasounds and it is reasonable to assume that similar results cannot be obtained by operators more or less experienced. The ultrasound is an examination operator-dependent. There was no consensus on the level of training required to acquire pulmonary ultrasound skills, and results may be different in other contexts because of different skills and experiences. Ultrasound learning is relatively simple and fast compared to other ultrasound applications [36-37].

\section{Conclusions}

Our study has shown that ultrasound is a sensitive and very specific tool in the service of Emergency Department for the diagnosis of pneumonia. It seems interesting to use it as first line examination in patients with suspected pneumonia. It could probably replace radiography and the use of ultrasound could give quick and accurate results so that the treatment be started early without having to wait for the biological and radiological results. However, further studies are needed to be done to evaluate the quality of the pulmonary ultrasound at the bedside for the diagnosis of pneumonia, taking into account the limitations of this study [38-39].

In addition, the training of young doctors in emergency ultrasound is not yet very widespread and depends on personal initiative. Finally, more studies with more patients are needed. Pulmonary ultrasound in emergency department, for the management of patients suspected of having pneumonia, could be an interesting tool and become the stethoscope of tomorrow.

\section{References}

1. Pinner RW, Teutsch SM, Simonsen L (1996) Trends in infectious diseases mortality in the United States. JAMA 275: 189-193.

2. Metlay JP, Fine MJ (2003) Testing strategies in the initial management of patients with community acquired pneumonia. Ann Intern Med 138: 109-118.

3. Hagaman JT, Rouan GW, Shipley RT, Panos RJ (2009) Admission chest radiograph lacks sensitivity in the diagnosis of community-acquired pneumonia. Am J Med Sci 337: $236-240$

4. Maughan BC, Asselin N, Carey JL, Sucov A, Valente JH (2014) False-negative chest radiographs in emergency department diagnosis of pneumonia. R I Med J 97: 20-23

5. Hayden GE, Wrenn KW (2009) Chest radiograph vs. computed tomography scan the evaluation of pneumonia. J Emerg Med 36: 266-270.

6. Syrjala H, Broas M, Suramo I, Ojala A, Lahde S (1998) High-resolution computed tomography for the diagnosis of community-acquired pneumonia. Clin Infect Dis 27:358-63.

7. Lichtenstein D, Mézière G, Biderman P (1997)The comet-tail artifact. An ultrasound sign of alveolar-interstitial syndrome. Am J Respir Crit Care Med 156: 1640-1646.
8. Ding W, Shen Y, Yang J (2011) Diagnosis of pneumothorax by radiography and ultrasonography: a meta-analysis. Chest 140: 859-866.

9. Cortellaro F, Colombo S, Coen D (2012) Lung ultrasound is an accurate tool for the diagnosis of pneumonia in the emergency department. Emerg Med J 29: 19-23.

10. Reissig A, Hevne JP, Kroegel C (2001) Sonography of lung and pleura in pulmonary embolism: sonomorphologic characterization and comparison with spiral CT scanning. Chest 120: 1977-1983.

11. ATS (2001) Guidelines for the management of adults with community-acquired pneumonia. Am J RespirCrit Care Med 163: 1730-1754.

12. BTS (2001) BTS guidelines for the management of community acquired pneumonia in adults. Thorax 56: 1-64.

13. Degelau J (2017) Community-acquired pneumonia in adults. Dtsch Arztebl Int 114 838-848.

14. Mandell L (2003) Update of practice guidelines for the management of communityacquired pneumonia in immunocompetent adults. Clin Infect Dis 37: 1405-1433.

15. Lichtenstein DA, Lascols N, Meziere G, Gepner A (2004) Ultrasound diagnosis of alveolar consolidation in the critically ill. Intensive Care Med 30: 276-281.

16. Lichtenstein DA, Mezière G, Lascols N, Biderman P, Courret JP, Gepner A (2005) Ultrasound diagnosis of occult pneumothorax. Crit Care Med 33: 1231-1238.

17. Volpicelli G, Mussa A, Garofalo G (2006) Bedside lung ultrasound in the assessment of alveolar interstitial syndrome. Am J Em Med 24: 689-696.

18. Lichtenstein D (2005) Lung ultrasound in the critically ill. Clin Intensive Care 16 79-87.

19. Ait-Ali L, Andreassi MG, Foffa I, Spadoni I, Vano E, Picano E (2010) Cumulative patient effective dose and acute radiation-induced chromosomal DNA damage in children with congenital heart disease. Heart 96: 269-274.

20. Andreassi MG (2009) Radiation risk from pediatric cardiac catheterization: friendly fire on children with congenital heart disease. Circulation 120: 1847-1849

21. Committee to Assess Health Risks from Exposure to Low Levels of Ionizing Radiation National Research Council. Health risks from exposure to low levels of ionizing radiation: BEIR VII Phase 2. 2006; National Academies Press: Washington, DC, USA.

22. Reissig A, Görg C, Mathis G (2009) Transthoracic sonography in the diagnosis of pulmonary diseases: a systematic approach. Ultraschall Med 30: 438-454.

23. Beckh S, Bölcskei PL, Lessnau KD (2002) Real-time chest ultrasonography: a comprehensive review for the pulmonologist. Chest 122: 1759-1773.

24. Lichtenstein D, Mezière G (2008) Relevance of lung ultrasound in the diagnosis of acute respiratory failure: the BLUE protocol Chest 134: 117-125

25. Sartori S, Tombesi P (2010) Emerging roles for transthoracic ultrasonography in pulmonarydiseases. World J Radiol 2: 203-214.

26. Agricola E, Bove T, Oppizzi M (2005) Ultrasound comet-tail images: a marker of pulmonary edema: a comparative study with wedge pressure and extravascular lung water. Chest 127: 1690-1695.

27. Lichtenstein D, Mezière G (1998) A lung ultrasound sign allowing bedside distinction between pulmonary edema and COPD: the comet-tail artifact. Intensive Care Med 24: $1331-1334$

28. Reissig A, Kroegel C (2003) Transthoracicsonography of diffuse parenchymal lung disease: the role of comet tail artifacts. J Ultrasound Med 22: 173-180.

29. Blaivas M, Lyon M, Duggal S (2005) A prospective comparison of supine chest radiography and bedside ultrasound for the diagnosis of traumatic pneumothorax. Acad Emerg Med 12: 844-849

30. Zhang M, Liu ZH, Yang JX (2006) Rapid detection of pneumothorax by ultrasonography in patients with multiple trauma. Crit Care 10: R112.

31. Hoffmann B, Gullett JP (2010) Bedside transthoracic sonography in suspected pulmonary embolism: a new tool for emergency physicians. Acad Emerg Med 17: 88-93.

32. Mathis G, Blank W, Reissig A (2005) Thoracic ultrasound for diagnosing pulmonary embolism: a prospective multicenter study of 352 patients. Chest 128: 1531-1538

33. Parlamento S, Copetti R, Di Bartolomeo S (2009) Evaluation of lung ultrasound for the diagnosis of pneumonia in the ED. Am J Emerg Med 27: 379-384

34. Lichtenstein D, Mezière G, Seitz J (2009) The dynamic air bronchogram. A lung ultrasound sign of alveolar consolidation ruling out atelectasis. Chest 135: 1421-1425. 
Citation: Daoulari N (2019) Comparison of Emergency Chest Ultrasound with Radiography in the Diagnosis of Community-acquired Pneumonia in Emergency Department. Journal of Medicine and Healthcare SRC/JMHC-106. DOI: doi.org/10.47363/JMHC/2019(1)104

35. Reissig A, Copetti R, Mathis G (2012) Lung ultrasound in the diagnosis and followup of community-acquired pneumonia. A prospective multicentre diagnostic accuracy study. Chest 142: 965-972.

36. Xiao-lei Liu, RuiLian, Yong-kang Tao, Cheng-dong Gu (2015) Guo-qiang Zhang Lung ultrasonography: an effective way to diagnose community-acquired pneumonia. Emerg Med J 32: 433-438.

37. Bedetti G, Gargani L, Corbisiero A, Frassi F, Poggianti E, Mottola G (2006) Evaluation of ultrasound lung comets by hand-held echocardiography. Cardiovasc Ultrasound 4: 34 .
38. Shah S, Noble VE, Umulisa I, Dushimiyimana JM, Bukhman G, Mukherjee J, Rich M, Epino H (2008) Development of an ultrasound training curriculum in a limited resource international setting: successes and challenges of ultrasound training in rural Rwanda. Int J EmergMed 1: 193-216.

39. Caiulo V, Gargani L, Caiulo S, Fisicaro A, Moramarco F, Latini G, Picano E, Melle G (2013) Lung ultrasound characteristics of community acquired pneumonia in Hospitalized Children, Pediatric Pulmonology 2012 Pediatr Pulmonol 48: 280-287.

Copyright: (C2021 Nelofer Baig, et al. This is an open-access article distributed under the terms of the Creative Commons Attribution License, which permits unrestricted use, distribution, and reproduction in any medium, provided the original author and source are credited. 